\title{
Comparison of Various Edge Detection Techniques in Tree Ring Structure
}

\author{
P.Meenakshi Sundari \\ Dept. of BCA, Fatima College, Madurai, Tamil \\ Nadu, Madurai
}

\author{
S.Britto Ramesh Kumar \\ Dept. of Computer Science, St. Joseph College, \\ Tiruchy, India
}

\begin{abstract}
The best result of image processing is depends on the fundamental steps, that is, to point out the true edges. Hence, it is very important to choose the edge detector, which fit best to the processing. This paper is a first step for analysing the rings in the tree ring image. In this paper we compare the various edge detection techniques towards the tree ring image and find out which fit best for further processing.
\end{abstract}

\section{Keywords}

Tree ring, sobel, prewitt, canny

\section{INTRODUCTION}

The study of tree growth layer and the past climate change in tree ring image is a tree-ring environment and climate science. Study of tree rings, help us to know about the dating of tree, find out the local climate etc[1]. Extracting the rings from the tree ring image is a major part of image processing. The growth of the tree is rapidly in spring,[2-4] resulting in the light colored early wood and the growth is decreased and become dark colored late wood in the late year. The dark colored late wood, look like a ring structure. Hence each year the dark ring structure is generated. Finding this late wood, that is, dark colored ring structure help us to understand the features of the tree and also its environment. Edge detecting techniques plays a vital role in finding the rings. The dark colored ring structures create a sharp change in the image brightness. The process of identifying and locating the sharp discontinuities in the image and abrupt changes in the pixel intensity is edge detection techniques. Edge detectors are a collection of very important local image pre-processing methods used to locate changes in the intensity function; edges are pixels where this function (brightness) changes abruptly. The image function depends on two variables, coordinates image plane and operators describing edges. Edge detectors are usually tuned for some type of edge profile. Various edge detection techniques are available. Canny, Sobel, prewit, Roberts etc. The operators examine small local neighbourhoods and detect edge direction by a collection of masks, each corresponding to a certain direction. techniques and analyze the performance of the various techniques in different conditions. There are many ways to perform edge detection. However, the majority of different methods may be grouped into two categories: Gradient based Edge Detection, Laplacian method. The gradient method detects the edges by looking for the maximum and minimum in the first derivative of the image. The Laplacian method searches for zero crossings in the second derivative of the image to find edges.

We ask that authors follow some simple guidelines. In essence, we ask you to make your paper look exactly like this document. The easiest way to do this is simply to download the template, and replace the content with your own material.

\section{PROBLEM DEFINITION}

Detecting the false edges or missing the true edges are some of the problems occur during the time of detecting the edges in the image. In this paper, apply various gradient and laplacian based edge detection techniques in tree ring images and analyzed the visual comparison between the techniques. Inaccurate edge detection, missing the true rings created a false report about the ring structure. Hence finding the edges in the tree ring image plays a major role for further analyzing.

\section{EDGE DETECTION TECHNIQUES}

The sharp variation in the intensity level is identified as an edge of the image. Before applying the image techniques, the image is converted into 8-bit type, that is, the intensity level of each pixel is 0 to 255 .

Consider the figure 1 tree ring image as an input image

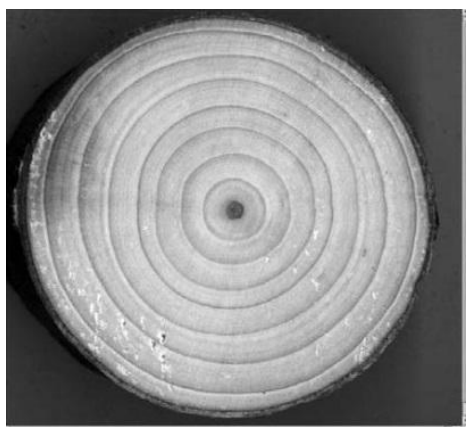

Fig 1 : Tree ring Input image

\section{Sobel Operator}

First we apply the sobel edge detection algorithm $[5,6]$. This algorithm uses four operator to measure the intensity variation in four direction: horizontal, vertical, left diagonal and right diagonal. The operator consists of a pair of $3 \times 3$ convolution kernels and rotated by $90^{\circ}$. Consider an input image of size mxn: The arrangement of pixel in the image,

Table 1: Arrangement of pixel in the image

\begin{tabular}{|c|c|c|c|c|}
\hline $\mathrm{A}(1,1)$ & $\mathrm{A}(1,2)$ & $\mathrm{A}(1,3)$ & $\ldots$ & $\mathrm{A}(1, \mathrm{n})$ \\
\hline $\mathrm{A}(2,1)$ & $\mathrm{A}(2,2)$ & $\mathrm{A}(2,3)$ & $\ldots$ & $\mathrm{A}(2, \mathrm{n})$ \\
\hline $\mathrm{A}(3,1)$ & $\mathrm{A}(3,2)$ & $\mathrm{A}(3,3)$ & $\ldots$ & $\mathrm{A}(3, \mathrm{n})$ \\
\hline & & & & \\
\hline $\mathrm{A}(\mathrm{m}, 1)$ & $\mathrm{A}(\mathrm{m}, 2)$ & $\mathrm{A}(\mathrm{m}, 3)$ & $\ldots$ & $\mathrm{A}(\mathrm{m}, \mathrm{n})$ \\
\hline
\end{tabular}

The algorithm uses four mask to the above $3 \times 3$ dimension selected from the above image. 


\begin{tabular}{|c|l|l|}
\hline \multicolumn{3}{|c|}{$\mathrm{H}$} \\
\hline 1 & 2 & 1 \\
\hline 0 & 0 & 0 \\
\hline-1 & -2 & -1 \\
\hline
\end{tabular}

(a)

\begin{tabular}{|c|c|c|}
\hline \multicolumn{3}{|c|}{ DL } \\
\hline 0 & 1 & 2 \\
\hline-1 & 0 & 1 \\
\hline-2 & -1 & 0 \\
\hline
\end{tabular}

(c)

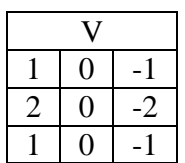

(b)

\begin{tabular}{|l|l|l|}
\multicolumn{3}{|c|}{ DR } \\
\hline 2 & 1 & 0 \\
\hline 1 & 0 & -1 \\
\hline 0 & -1 & -2 \\
\hline
\end{tabular}

(d)
Table 2: (a)Horizontal detection (b)Vertical (c) Diagonal Left (d) Diagonal Right

To obtain the value of the intensity variation, the following functions are used:

$\mathrm{Eh}=(\mathrm{A}(1,1)+2 \mathrm{~A}(1,2)+\mathrm{A}(1,3))-(\mathrm{A}(3,1)+2 \mathrm{~A}(3,2)+\mathrm{A}(3,1))$

$\mathrm{Ev}=(\mathrm{A}(1,1)+2 \mathrm{~A}(2,1)+\mathrm{A}(3,1))-(\mathrm{A}(1,3)+2 \mathrm{~A}(2,3)+\mathrm{A}(3,3))$

$\mathrm{Edl}=(\mathrm{A}(1,2)+2 \mathrm{~A}(1,3)+\mathrm{A}(2,3))-(\mathrm{A}(2,1)+2 \mathrm{~A}(3,1)+\mathrm{A}(3,2))$

$\mathrm{Edr}=(2 \mathrm{~A}(1,1)+\mathrm{A}(1,2)+\mathrm{A}(2,1))-(\mathrm{A}(2,1)+\mathrm{A}(3,2)+2 \mathrm{~A}(3,3))$

After finding the values in each direction, the gradient magnitude and the direction of each variation is computed.

Magnitude $=\operatorname{Max}[|E h|,|E v|,|E d l|,|E d r|]+1 / 8\left[\left|E_{p e r}\right|\right]$

$\mathrm{E}_{\mathrm{per}}$ is a $\mathrm{n}$ intensity variation perpendicular to the maximum intensity variation. The magnitude value has to be compared with a signal of the edge detector code called threshold. If the magnitude is greater than the threshold, the value 255 is assigned to the correspondent pixel otherwise 0 .

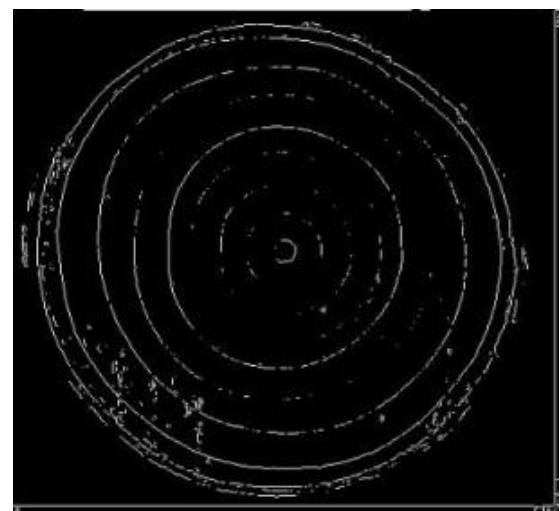

Fig 2: Applying sobel operator in figure 1

The Prewitt edge detector:

This detector $[5,6]$ is used to detect the edges in the horizontal and vertical sequence

The arrangement of pixel in the image is as in table 1.

Select $3 \times 3$ dimensions from the table.

\begin{tabular}{|l|l|l|}
\hline-1 & 0 & 1 \\
\hline-1 & 0 & 1 \\
\hline-1 & 0 & 1 \\
\hline
\end{tabular}

\begin{tabular}{|c|c|c|}
\hline-1 & -1 & -1 \\
\hline 0 & 0 & 0 \\
\hline 1 & 1 & 1 \\
\hline
\end{tabular}

Table 3: Horizontal filter, Vertical filter of prewitt edge detection

The intensity value for each pixel is calculated by

pixel $=(\mathrm{A}(1,3)+\mathrm{A}(3,2)+\mathrm{A}(3,3))-(\mathrm{A}(1,1)+\mathrm{A}(2,1)+\mathrm{A}(3,1)))+$ $((\mathrm{A}(3,1)+\mathrm{A}(3,2)+\mathrm{A}(3,3)-(\mathrm{A}(1,1)+\mathrm{A}(1,2)+\mathrm{A}(1,3)))$

The intensity value of each pixel gives the value from 0-255.

Applying the prewitt edge detector, the following output image appeared.

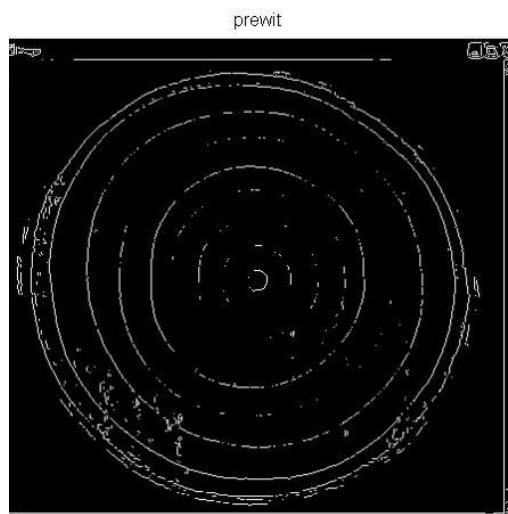

Fig 3: Applying prewitt operator in figure 1 Roberts edge detector

This is [5,6]simple and fast compute of 2-D spatial gradient measurement of on the image. This detector extract the high spatial gradient from the image. The 2 x 2 convolution masks are:

\begin{tabular}{|c|c|}
\multicolumn{2}{|c|}{$\mathrm{Gx}=$} \\
\hline 1 & 0 \\
\hline 0 & -1 \\
\hline
\end{tabular}

$$
\mathrm{Gy}=
$$

\begin{tabular}{|c|c|}
\hline 0 & 1 \\
\hline-1 & 0 \\
\hline
\end{tabular}

Table 4: spatial gradient for Roberts edge detector

This mask responds to the edges running at 45 degree to the pixel grid.

The magnitude of each pixel corresponding to the table 1 is

$$
\mathrm{Mag}=(|\mathrm{A}(1,1)-\mathrm{A}(2,2)|+|\mathrm{A}(1,2)-\mathrm{A}(2,1)|)
$$

Applying the roberts edge detector to the tree ring image, the resulted image as follows

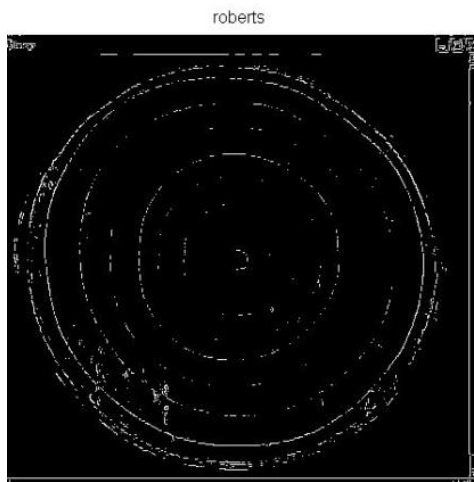

Fig 4: Applying Roberts operator in figure 1 Canny Edge detection techniques 
This is technique [5,6] is an optimal detector of edge in tree ring image. It takes the gray scale image of tree ring as an input and produces the output showing the tracked intensity discontinuity. This paper followed a list of criteria to improve in detecting edges

- Low error rate, edges should not be missed and also not response to non-edge.

- The points in the edge should be well localized.

- Response to a single edge. The first two criteria is not enough to eliminate the response for multiple edges.

Canny edge algorithm uses four filters to detect the edges, because an edge may point in a variety of direction. Compute the derivatives $(\operatorname{Dx}(\mathrm{x}, \mathrm{y})$ and $\operatorname{Dy}(\mathrm{x}, \mathrm{y}))$ of the image in the $\mathrm{x}$ and $y$ directions. Then compute the magnitude similar to sobel operator

\begin{tabular}{|l|l|l|}
\hline \multicolumn{3}{|c|}{$\mathrm{Dy}(\mathrm{x}, \mathrm{y})$} \\
\hline 1 & 2 & 1 \\
\hline 0 & 0 & 0 \\
\hline-1 & -2 & -1 \\
\hline
\end{tabular}

(a)

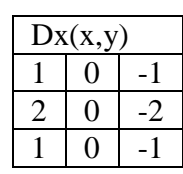

(b)
Table 4 : a) Dy(x,y)Horizontal filter, b) Dx(x,y) Vertical filter

The angle of the gradient is

$$
\theta=\arctan (D x(x, y) / D y(x, y))
$$

The $\theta$ is computer by rounding the angleto one of four directions $0^{\circ},, 45^{\circ}, 90^{\circ}$, or $135^{\circ}$, and for $180^{\circ}=0^{\circ}, 225^{\circ}=$ $45^{\circ}$. This means $\theta$ in the ranges $\left[-22.5^{\circ} \ldots 22.5^{\circ}\right]$ and $\left[157.5^{\circ} \ldots 202.5^{\circ}\right]$ would "round" to $\theta^{\prime}=0$ 。

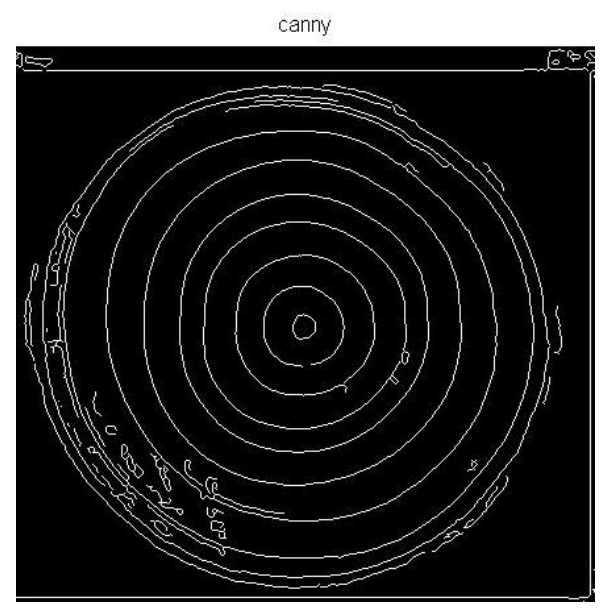

Fig 5: Result of canny edge detection

\section{ADVANTAGES \& DISADVANTAGES OF EDGE DETECTOR}

In the previous chapter, we discussed the application of various edge detection methods for tree ring analyses.
Concluding that chapter, here list out the advantage and disadvantage of various edge techniques.[7]

\section{Sobel, Prewitt Operator, Roberts:}

The advantage: Simple in implementation of magnitude and the orientation.

Disadvantage: Sensitivity to noise and cannot give best result of detecting the rings.

\section{Canny edge detection}

Advantage: Low error rate, the edge should not missed, eliminate the duplicate edges, localization and response, better edge detection, especially in the noise condition.

Disadvantage: Complex computation.

Comparing all the result of various edge detections, the canny technique is more suitable for the first step of detecting rings in the tree ring detection.

\section{CONCLUSION}

It is necessary to know the difference between various edge detection methods because finding the edge is the initial step for processing the tree ring image. The gradient based algorithm such as prewitt filter have major drawback over the noise signal. The performance of canny edge algorithm is improved by adjusting the parameter. It is computationally more expensive compared to sobel, prewitt and Roberts operator. But this algorithm performs better than all these operators in extracting the rings in the image. Improvising canny edge detection algorithm for getting better result to all the types tree ring images.

\section{REFERENCES}

[1] Hang-jun Wang, Guang-qun Zhang, Heng-nian Qi,Wenzhu Li, Bi-hui Wang, "An Automatic Method of TreeRings Boundary Detection on Wood Microimages",ICCASM 2010.

[2] Qinhua Tian, Samuli Helama, Ping Zhao, "Temperature Variablity inferred from Tree Rings of Qiniling Region in North-Central China", 2011, IEEE(2072 -2075)

[3] Amalava Bhattacharyya, Santosh K. Shah, "Tree-Ring Studies in India Past Appraisal, Present Status and Future prospects", IAWA Journal, Vol. 30 (4), 2009:361-370.

[4] Yinke YANG, Qiang HUANG, Wenke WANG, Yu LIU, Yimin WANG, Hongbin YANG, "Experimental Methods on Tree-Ring Density of Dendrochronology", 2011, IEEE, 342-345.

[5] Anil.K.Jain, Fundamentals Of Digital Image Processing, Prentice Hall,Englewood Cliffs, New Jersey 07632.

[6] Rafael C.Gonzalez, Richard E.Woods, Digital Image Processing, third edition, Prentice Hall, Chapter 10.

[7] G.T. Shrivakshan1, Dr.C. Chandrasekar, A Comparison of various Edge Detection Techniques used in Image Processing, IJCSI International Journal of Computer Science Issues, Vol. 9, Issue 5, No 1, September 2012 